\title{
PROPIEDADES FÍSICAS Y MECÁNICAS DE UN CONTRACHAPADO DE COIGÜE FABRICADO EN CHILE.(*)
}

\author{
VICENTE PÉREZ GALAZ (**)
}

(*) Proyecto FONDECYT N 1950295 - 1995

(**) Ingeniero Civil , U. de Chile, Profesor Asociado, Departamento de Ingeniería en Obras Civiles, Facultad de Ingenieria. Universidad de Santiago de Chile, Avda. B. O'Higgins 3363. Santiago - Chile

\section{RESUMEN}

El presente trabajo determina las propiedades fisicas y mecánicas de un contrachapado estructural de coigue (Nothofagus dombey Mirh. Blume) fabricado en Chile.

El objetivo del estudio es conocer las Tensiones Admisibles de dicho contrachapado estructural a fin de incrementar el pequeño banco de datos que sobre el tema existe en el pais y contar asi los elementos que permitan realizar el cálculo estructural de elementos constructivos que incluyan a dicho material como parte constitutiva de vigas compuestas, paneles de muro, de cielo, y de piso, pilares compuestos, pisos, muros, cielos, arcos, marcos, etc.

El articulo revisa las caracteristicas que debe tener el contrachapado estructural y describe el procedimiento de muestreo y posterior ensavo de las probetas extraidas de los tableros muestreados.

Las conclusiones establecen que el material usado tenia: Un contenido de humedad promedio de $9.1 \%$, densidades de referencia de $483 ; 523$ y $650 \mathrm{~kg} / \mathrm{m}^{3}$ para espesores de $9.12 \mathrm{y}$ $15 \mathrm{~mm}$, respectivamente; $y$ pesos por unidad de superficie de $4,: 6.6$ y $10 \mathrm{~kg} / \mathrm{m}^{2}$ para dichos espesores.

Las propiedades mecánicas se determinan con los tres criterios que existen para obtener las propiedades geométricas, a saber: La sección transversal bruta. la sección transversal efectiva y la seccion transformada.

Se entregan, además, las Tensiones Admisibles calculadas con estos tres métodos y finalmente se establece que el contrachapado estructural de coigüe es comparable con la Clase Estructural F 14 , que entrega la norma chilena $\mathrm{NCh} 1198$.

Palabras Clave: Madera, Contrachapado, Propiedades. 


\section{ABSTRACT}

The physical and mechanical properties of coigüe plywood manufactured in Chile are shown. The main characteristics of the plywood are analyzed. Sampling and testing procedures of the specimens extracted from the plywood boards are described.

The main objective of the study was to determine the basic working stresses of the structural plywood in order to increase the chilean data base on the subject and the use of this material in the design of composite beams, walls, ceilings, floors, composite columns, arches, structural frames, etc.

The material was characterized as follows: average moisture content $9,1 \%$, bulk densities of 483: 523 and $650 \mathrm{~kg} / \mathrm{m}^{3}$ for the thicknesses of 9,12 and $15 \mathrm{~mm}$ respectively, and weight per unit area of 4.7:6.6 and $10 \mathrm{~kg} / \mathrm{m}^{2}$ for the same.

Mechanical properties were determined using the three existing criteria: bulk cross section. effective cross section and transformed cross section. Basic working stresses using the three criteria were obtained. Finally it is established that coigüe structural plywood meets structural class Fl4 of the chilean code NCh 1198.

Keywords: Wood, Plywood, Properties. 


\section{INTRODUCCIÓN}

La madera, pese a ser uno de los materiales de construcción más antiguos con que cuenta la humanidad, mantiene su vigencia compitiendo exitosamente con materiales modernos y revolucionarios, como son aceros, hormigones y plásticos. Para ello, el hombre ha necesitado desarrollar nuevas técnicas de aprovechamiento de las maderas naturales con el objeto de obviar sus limitaciones físicas y dimensionales. Entre los productos de estas nuevas técnicas se encuentran los "tableros contrachapados"

Por tablero se entiende un elemento largo, ancho y delgado obtenido de la madera por medio de algún proceso industrial, a veces bastante complejo. Se utilizan para fines estructurales o decorativos, y se distinguen: los de madera contrachapada, los de fibra y los de partículas.

Para muchas aplicaciones los tableros presentan ventajas considerables respecto de la madera aserrada. Entre éstas pueden citarse las siguientes: uniformidad en la calidad. estabilidad dimensional, facilidad con que se puede trabajar con técnicas y herramientas sencillas, superficies de dimensiones amplias, facilidad con que pueden aplicarse acabados diversos y sencillez de aplicación. Además, en muchos casos sus propiedades resistentes (mecánicas) son superiores a las de la madera aserrada. Los tableros de madera tienen múltiples aplicaciones, entre Jas que destacan moldajes o encofrados. elementos estructurales o de terminación para viviendas, muebles, embalajes y embarcaciones.

Las ventajas que ofrecen han hecho que el consumo mundial de tableros haya aumentado más rápidamente que el de cualquier otro tipo de producto de madera, el incremento que ha sido más notable en las regiones donde la madera aserrada es cara o escasa. Así, en la actualidad, el consumo de tableros por habitante en los países desarrollados es de quince a veinte veces superior al de América Latina. Sin embargo. para el futuro, se estima que el consumo empezará a estabilizarse en Europa y Norteamérica, mientras se espera que en América Latina habrá un incremento gradual.

Lạ madera contrachapada está constituída por tres o más hojas o "tulipas" unidas por una sustancia adhesiva y dispuestas de tal forma que las fibras de cada chapa estén perpendiculares respecto de las mismas de las chapas contiguas. Para evitar alabeos por falta de simetría el número de láminas generalmente es impar, colocándose pares de chapas a los lados de una hoja central, de tal manera que para cada lámina exista una opuesta, similar y paralela.

Las "tulipas" para fabricar el tablero contrachapado se obtienen "desenrrollándolas" con un gran cuchillo, de trozas que se hacen girar sobre su eje. Además, se pueden obtener chapas mediante una guillotina o cuchilla que se desliza paralelamente a la superficie de corte del tronco, cuadrado o tablón, sin que haya giro. Las máquinas 
usadas inicialmente para la fabricación del contrachapado requerían trozas de gran diámetro, buena calidad y forma regular. En la actualidad se han desarrollado procedimientos y productos que permiten aprovechar trozas de diámetro pequeño y calidad inferior.

La madera contrachapada puede fabricarse de diversos tipos decorativos y estructurales, variando el número y espesor de las chapas, la especie y calidad de la madera de las diversas capas, el acabado superficial y el tipo de adhesivo usado. Uno de los de mayor utilidad en la construcción es el contrachapado estructural.

Gracias a su constitución a base de chapas dispuestas de manera que las fibras de cada una queden perpendiculares a las de las tulipas contiguas, se mitiga uno de los inconvenientes de la madera : su anisotropía. En efecto, las resistencias del tablero contrachapado en sus dos direcciones principales son semejantes. Otras ventajas que se derivan de la estructura peculiar del contrachapado son su alta resistencia a la extracción lateral de clavos, pernos y tornillos, su estabilidad dimensional y su alta resistencia a los esfuerzos de corte que se originan en su plano. Esta última propiedad es ventajosa en el diseño de diafragmas y otros elementos estructurales semejantes. Por otra parte, el poco peso de los tableros y los componentes estructurales de contrachapado. así como la facilidad con que se ensamblan, permiten una notable rapidez en los procesos de construcción, sin necesidad de utilizar equipo pesado. De especial interés es la posibilidad de recurrir a sistemas de prefabricación para producir componentes estándares para muros, techumbres y pisos. Estos componentes. que pueden fabricarse en instalaciones sencillas que no requieren grandes inversiones. parecen ofrecer un gran potencial para la construcción de viviendas.

Como lo demuestra la experiencia de otros países, la madera contrachapada ofrece un sinnúmero de posibilidades y ventajas para la construcción. Una de las razones por las que su uso en Chile está restringido a aplicaciones no estructurales o estructuras de diseño empírico, como suele suceder con los moldajes, es la falta de información adecuada sobre las propiedades físicas y mecánicas del contrachapado disponible. Influye también la escasa experiencia de los calculistas para diseñar elementos estructurales de contrachapados. El uso racional y económico de estos tableros, de manera que su empleo sea el más apropiado según su función estructural y las condiciones de humedad a la que vayan a estar expuestos, requiere de un conocimiento adecuado y confiable de sus propiedades mecánicas y de la resistencia a la humedad de los adhesivos empleados, el desarrollo de métodos de diseño prácticos, y de una intensa labor de divulgación entre profesionales y técnicos.

En Chile se conocen las propiedades del contrachapado constituido de tulipas de tepa-olivillo, y de pino radiata. El objetivo del presente trabajo es agregar, a los datos anteriores. las propiedades del contrachapado estructural fabricado con chapas de coigüe (Nothofagus dombeyi Mirb. Blume) por la industria BOMASA. 


\section{REQUISITOS GENERALES DEL CONTRACHAPADO ESTRUCTURAL}

Como no existen normas nacionales sobre la materia, se decidió adoptar el criterio establecido por las normas australianas para fijar los requisitos que debe cumplir el contrachapado estructural. Ellas señalan:

- El contrachapado estructural debe ser clasificado de acuerdo a la calidad, por apariencia, de las superficies de las caras exteriores ( AS 2269)

- Las superficies de las caras exteriores del contrachapado estructural pueden ser: GRADO C y GRADO D y deben corresponder, respectivamente, a chapas de calidad C y calidad D.

En una publicación anterior (Pérez, 1995), se incluyeron las especificaciones de los distintos grados de calidad, por apariencia, de las chapas.

En cuanto al tipo de adhesivo, la norma australiana AS 2269 señala:

- Los contrachapados estructurales deben tener una unión TIPO A. usando un adhesivo a base de fenol-formaldehido.

- Los tableros de contrachapado estructural, fabricados con chapas exteriores de CALIDAD C y/o CALIDAD D y encolados con un TIPO A de unión, deben ser clasificados estructuralmente por uno de los dos procedimientos siguientes :

i) Método de la Densidad de las chapas,

ii) Método de Clasificación Mecánica.

El resultado que entregan estos métodos es la identificación de la Clase o Grado Estructural del tablero en estudio. Con dicho Grado Estructural se obtienen las Tensiones Admisibles aplicables al tablero.

Cada tablero de contrachapado estructural debe contener la siguiente información. en forma legible e indeleble, por lo menos una vez.

a) Nombre del fabricante o la marca registrada.

b) La palabra "ESTRUCTURAL".

c) El grado del aspecto de las chapas exteriores (la cara primero y luego la trascara) Ejemplo : CC, CD ó DD.

d) El tipo de encolado aplicado entre chapas. Ejemplo: UNION : TIPO A.

e) Una indicación del Grado Estructural del tablero. Ejemplo: F7 ó una marca con el color apropiado. 
- Los tableros deben cumplir, además, con las especificaciones que entregan las normas relacionadas con contenido de humedad, procesos constructivos, dimensiones, tolerancias y formas. Como tales especificaciones ya fueron entregadas en la publicación anterior (Pérez, 1995), no se incluirán en éste documento.

\section{PROPIEDADES FISICAS Y MECÁNICAS ESTUDIADAS}

Las propiedades fisicas estudiadas para el tablero contrachapado estructural de coigüe, fueron :

- Densidad de Referencia y Densidad Nominal, en $\mathrm{kg} / \mathrm{m}^{3}$.

- Contenido de Humedad, en \%

- Peso por unidad de superficie, en $\mathrm{kg} / \mathrm{m}^{2}$.

Las propiedades mecánicas determinadas para el tablero contrachapado estructural de coigüe, fueron:

- Flexión Estática con carga aplicada en la cara del tablero.

- Tensión en el límite de proporcionalidad.

- Módulo de Ruptura.

- Módulo de Elasticidad.

- Flexión Estática con carga aplicada en el canto.

- Tensión en el limite de proporcionalidad.

- Módulo de Ruptura.

- Módulo de Elasticidad.

- Compresión para carga paralela a las fibras de las caras exteriores.

- Tensión Máxima.

- Compresión para carga perpendicular a las fibras de las caras exteriores.

- Tensión Máxima.

- Tracción para carga paralela a las fibras de las caras exteriores.

- Tensión Máxima.

- Tracción para carga perpendicular a las fibras de las caras exteriores.

- Tensión Máxima. 
- Cizalle a través del espesor, para carga aplicada en el canto. con dirección paralela a las fibras de la cara exterior.

- Tensión Máxima.

- Cizalle a través del espesor, para carga aplicada en el canto, con dirección perpendicular a las fibras de la cara exterior.

- Tensión Máxima.

- Cizalle entre chapas, para carga aplicada en el canto, con dirección perpendicular a las fibras de la cara exterior.

- Tensión Máxima.

- Módulo de cizalle en el plano del tablero ( G )

Todos los ensayos enumerados siguieron las especificaciones de las Normas de la AMERICAN SOCIETY FOR TESTING MATERIALS (ASTM), con excepción del ensavo de cizalle a través del espesor. para el cual se siguió la recomendación de los investigadores alemanes Möhler y Ehlbeck (1974).

\section{METODOLOGÍA DE EXPERIMENTACIÓN}

\section{Material Usado}

El material se seleccionó de las bodegas de BOMASA de tal manera que la calidad de las chapas ubicadas en las caras exteriores cumplieran con los requisitos de la norma australiana AS 2269. Además, se comprobó que el adhesivo usado fuese a base de fenol-formaldehido. Se postergó la clasificación estructural hasta el momento en que se havan obtenido todas las propiedades físicas y mecánicas de los tableros contrachapados seleccionados.

Los tableros contrachapados usados para extraer las distintas probetas que especifican las normas ASTM. tenian las siguientes características:
Especie
1 (Coigüe)
Dimensiones del tablero
$1(1220 * 2440 \mathrm{~mm})$
Espesores nominales
$3(9 \mathrm{~mm}, 12 \mathrm{~mm}$ y $15 \mathrm{~mm})$
Repeticiones de tableros
: 6
TOTAL
18 tableros 


\section{Metodología De Ensayo}

Se describe, a continuación, la metodología de ensayo señalando las distintas etapas que se siguieron desde que los tableros ingresaron al laboratorio hasta que se procedió con el ensayo de las probetas.

Determinación de las Características Físicas de cada Tablero

A cada tablero se le controló sus dimensiones (espesor, ancho, largo) y su ortogonalidad. Se determinó, además, su peso, número de chapas que lo constituían y espesor de cada una de tales chapas. Estas medidas permitieron conocer el volumen de cada tablero y su densidad referencial.

Marcado y Obtención de las Probetas

Sobre la superficie de los tableros y con ayuda de una plantilla se marcaron las diferentes probetas, conservando la distribución de ellas en los distintos tableros. La identificación adoptada fue la siguiente:

$$
\mathrm{V}-\mathrm{W}-\mathrm{X}-\mathrm{Y}-\mathrm{Z}
$$

en que:

$$
\begin{array}{rll}
\mathrm{V}: & \text { FCAR } & =\text { Flexión con carga en la cara. } \\
: & \text { FCAN } & =\text { Flexión con carga en el canto. } \\
: & \mathrm{C} & =\text { Compresión. } \\
: & \mathrm{T} & =\text { Tracción. } \\
: & \mathrm{CZES} & =\text { Cizalle a través del espesor. } \\
: & \mathrm{CZIL} & =\text { Cizalle interlaminar. } \\
: & \mathrm{G} & =\text { Módulo de Cizalle en el Plano. } \\
\mathrm{W}: & \mathrm{P} & =\text { Carga paralela a fibra cara exterior. } \\
: & \mathrm{N} & =\text { Carga normal a fibra cara exterior. } \\
\mathrm{X}: & \mathrm{E} 1 & =\text { Espesor del tablero : } 9 \mathrm{~mm} . \\
: & \mathrm{E} 2 & =\text { Espesor del tablero : } 12 \mathrm{~mm} . \\
: & \mathrm{E} 3 & =\text { Espesor del tablero }: 15 \mathrm{~mm} . \\
\mathrm{Y}: & \mathrm{T} 1 & =\text { Tablero } 1 \mathrm{~A} \text { y Tablero } 1 \mathrm{~B} . \\
: & \mathrm{T} 2 & =\text { Tablero } 2 \mathrm{~A} \text { y Tablero } 2 \mathrm{~B} . \\
: & \mathrm{T} 3 & =\text { Tablero } 3 \mathrm{~A} \text { y Tablero } 3 \mathrm{~B} .
\end{array}
$$




\section{Z : $\quad$ Probeta, repetición $1,2, \ldots .5$.}

De esta forma la probeta identificada con la expresión:

$$
\text { FCAR - N - E2 - T3 - } 3 \text {. }
$$

significa que se trata de la probeta sometida a Flexión, con carga aplicada en la cara de dirección perpendicular a las fibras de su cara exterior. cuyo espesor es $12 \mathrm{~mm}$, proveniente del Tablero 3, repetición $\mathrm{N}^{\circ} 3$.

En todos los ensayos es necesario aplicar cargas y, en la mayoria de ellos, se requiere conocer además la deformación que se origina en la probeta para cada carga aplicada. La carga fue ejercida mediante una bomba hidráulica manual que fue conectada a un gato hidráulico. Para medir la deformación se instaló un dial, apoyado en la probeta, bajo el punto de aplicación de la carga.

Para la lectura de la carga y la deformación se ocupa un sistema computacional mediante el uso de un programa denominado Labtech, con el cual es posible recibir señales eléctricas que son leídas por el computador y archivadas en forma inmediata.

Para medir la carga se instala una celda de carga bajo el gato hidráulico de tal manera que al ser aplicada la celda envie una señal (en milivolts) al computador. Para la deformación. el sistema consiste en una vaina hueca por la cual se desliza una varilla metálica que al descender genera una diferencia de potencial (volts), que también es leída por el computador. Dado que en el tiempo cero existe una deformación y una carga inicial, es necesario calibrar el computador con estos valores. La conexión del dial de deformación y la celda de carga al computador se realiza a través de una interfase. Para facilitar el trabajo, es posible transformar las unidades eléctricas obtenidas en unidades de carga y deformación, por ejemplo: $\mathrm{kg}-\mathrm{f}$ y $\mathrm{mm}$, usando un factor de escala entregado junto con el programa computacional. De esta manera los datos que se archivan quedan en $\mathrm{Kg}-\mathrm{f}$ y $\mathrm{mm}$. Además, se requieren de ciertos parámetros concernientes al formato de presentación y captación de datos. Es decir se ingresa la duración del ensayo. los canales abiertos para la entrada de la información. el nombre del archivo en el que se guardarán los datos y los valores iniciales de carga y deformación.

Con los datos así ingresados en la pantalla y al hacer correr el programa, éste muestra dos gráficos: Carga - Tiempo y Deformación - Tiempo. Paralelamente se está generando un archivo, que como ya se explicó, tiene un formato numérico que puede ser leído en una pantalla electrónica.

Todos los ensayos ejecutados en la fase experimental se realizaron en el Laboratorio de Estructuras del Departamento de Ingeniería en Obras Civiles de la Facultad de Ingenieria. Universidad de Santiago de Chile (USACH). El equipo principal de esta fase consistió en un Marco Universal de Ensayo. metálico, el cual fue financiado con aportes de FONDECYT y de la USACH. 
El objetivo de la fase experimental es la obtención y tabulación de los datos que entregan los distintos ensayos de las 612 probetas contempladas en el presente estudio, con el fin de calcular las propiedades mecánicas (tensiones en el límite de proporcionalidad. las tensiones máximas, el módulo de elasticidad y el módulo de cizalle en el plano del tablero (G)) de los diferentes espesores de tableros seleccionados, lo cual constituye la meta final de este trabajo.

El hecho de incluir el computador como herramienta de experimentación, permite obtener una mayor cantidad de datos y, por lo tanto. analizar con mayor precisión cada ensayo. Otra ventaja es la posibilidad de medir las deformaciones que se producen al momento en que la probeta alcanza la ruptura. lo cual no es siempre posible con el método tradicional que implicaba el uso de un dial mecánico.

\section{RESULTADOS OBTENIDOS}

\section{Contenido de Humedad.}

El contenido de humedad de los tableros se determinó en muestras extraidas de los trozos de probetas ensayadas que provenian de un mismo tablero. Tal determinación se realizó por el método de secado en estufa y en el cálculo de la humedad se utilizó la expresión:

$$
H=\frac{P_{o}}{P_{s}} * 100
$$

en que

$$
\begin{aligned}
& \text { Po }=\text { peso original de la muestra después del ensayo, } g \text {. } \\
& \text { Ps }=\text { peso anhidro de la muestra después del ensayo. } g \text {. }
\end{aligned}
$$

En el Cuadro $\mathrm{N}^{\circ} 1$ se incluyen los valores medios que resultaron con el procedimiento antes descrito: 


\section{INSTITUTO FORESTAL}

\section{Cuadro $\mathrm{N}^{\mathrm{O}} 1$}

CONTENIDO DE HUMEDAD DE LOS TABLEROS

\begin{tabular}{|c|c|c|}
\hline $\begin{array}{c}\text { ESPESOR } \\
\text { NOMINAL } \\
(\mathrm{mm}) \\
\end{array}$ & $\begin{array}{l}\text { CANTIDAD DE TABLEROS } \\
\text { ENSAYADOS } \\
\text { (No) }\end{array}$ & $\begin{array}{c}\text { CONTENIDO } \\
\text { DE HUMEDAD } \\
\text { PROMEDIO } \\
(\%) \\
\end{array}$ \\
\hline 9 & 3 & 8,7 \\
\hline 12 & 3 & 9,0 \\
\hline 15 & 3 & 9,7 \\
\hline
\end{tabular}

\section{Densidad De Los Tableros.}

La densidad de los tableros se determinó en las muestras ocupadas para la determinación del contenido de humedad, usándose las siguientes expresiones:

$$
\begin{array}{lll}
\text { Densidad de Referencia: } & \rho_{r}=\frac{P_{o}}{V_{o}} & \left(\mathrm{~g} / \mathrm{cm}^{3}\right) \\
\text { Densidad Básica: } & \rho_{n}=\frac{P_{s}}{V_{o}} & \left(\mathrm{~g} / \mathrm{cm}^{3}\right)
\end{array}
$$

en que:

Po $=$ peso original de la muestra antes del ensayo, $g$.

Ps $=$ peso anhidro de la muestra después del ensayo, $g$.

$V_{0}=$ volumen original de la muestra antes del ensayo, $\mathrm{cm}^{3}$.

En el Cuadro $\mathrm{N}^{\circ} 2$ se incluyen los valores promedios de ambos tipos de densidades. en $\mathrm{kg} / \mathrm{m}^{3}$.

\section{Cuadro $\mathrm{N}^{\circ} 2$}

DENSIDAD DE IOS TABLEROS

\begin{tabular}{|c|c|c|c|}
\hline $\begin{array}{c}\text { ESPESOR } \\
\text { NOMINAL }\end{array}$ & $\begin{array}{c}\text { CANTIDAD DE } \\
\text { TABLEROS }\end{array}$ & $\begin{array}{c}\text { DENSIDAD PROMEDIO } \\
\left(\mathrm{kg} / \mathbf{m}^{3}\right)^{3}\end{array}$ \\
\cline { 3 - 4 } & $\left(\mathrm{N}^{\circ}\right.$ & REFERENCIA & BASICA \\
\hline 9 & 3 & 483,3 & 441,1 \\
\hline 12 & 3 & 523,4 & 476,5 \\
\hline 15 & 3 & 649,9 & 586,8 \\
\hline
\end{tabular}




\section{Peso por Unidad de Superficie}

El peso por unidad de superficie se obtuvo con los datos determinado: en los distintos tableros usados, a saber: su peso y las dimensiones del plano del tablero. Los resultados de este procedimiento se incluyen en el Cuadro $\mathrm{N}^{\mathrm{3}} 3$.

\section{Cuadro $N^{\circ} 3$.}

PESO POR UNIDAD DE SUPERFICIE DE LOS TABLEROS.

\begin{tabular}{|c|c|c|}
\hline $\begin{array}{c}\text { ESPESOR } \\
\text { NOMINAL } \\
(\mathrm{mm})\end{array}$ & $\begin{array}{c}\text { CANTIDAD DE } \\
\text { TABLEROS } \\
\left(\mathrm{N}^{\circ}\right)\end{array}$ & $\begin{array}{c}\text { PESO POR UNIDAD DE } \\
\text { SUPERFICIE } \\
\left(\mathrm{kg} / \mathrm{m}^{2}\right)\end{array}$ \\
\hline 9 & 3 & 4,72 \\
\hline 12 & 3 & 6.55 \\
\hline 15 & 3 & 9,97 \\
\hline
\end{tabular}

\section{Flexión con Carga Aplicada sobre la Cara}

Los resultados que se entregan para este ensayo están calculados con la seccion transversal efectiva. es decir considerando como secciones efectivas de una sección transversal. sólo a las chapas cuyas fibras tienen la misma dirección de los esfuerzos principales. o dicho de otra forma. a las chapas que tienen sus fibras con dirección paralela a la luz de la viga.

Los valores señalados se incluyen en los Cuadros $\mathrm{N}^{\circ}+y \mathrm{~N}^{\circ} 5$

\section{Cuadro $\mathrm{N}^{0} 4$}

RESULTADOS DEL ENSAYO DE FLEXIÓN ESTÁTICA CON CARGA APLICADA EN LA CARA. DIRECCIÓN DE LAS FIBRAS DE CARAS EXTERIORES, PARAI,ELA A LA LUZ.

\begin{tabular}{|c|c|c|c|c|}
\hline $\begin{array}{c}\text { ESPESOR } \\
\text { NOMINAL } \\
(\mathbf{m m}) \\
\end{array}$ & $\begin{array}{c}\text { ESTADIGRAFOS } \\
(-)\end{array}$ & $\begin{array}{c}\text { TENSION EN EL } \\
\text { LIMITE DE } \\
\text { PROPORCIONALIDAD } \\
\left(\mathrm{kg} / \mathrm{cm}^{2}\right)\end{array}$ & $\begin{array}{l}\text { MÓDULO DE } \\
\text { RUPTURA } \\
(\mathrm{kg} / \mathrm{cm} 2)\end{array}$ & $\begin{array}{c}\text { MÓDULO DE } \\
\text { ELASTICIDAD } \\
\left(v \mathrm{~cm}^{2}\right)\end{array}$ \\
\hline 9 & $\begin{array}{l}\text { Promedio } \\
\text { D. Estándar } \\
\text { C. de Variac. } \\
\text { N }\end{array}$ & $\begin{array}{c}796,7 \\
68,15 \\
8.6 \% \\
14\end{array}$ & $\begin{array}{c}960.9 \\
181.73 \\
18.9 \\
14\end{array}$ & $\begin{array}{c}132.7 \\
12,44 \\
9.4 \% \\
14\end{array}$ \\
\hline 12 & $\begin{array}{l}\text { Promedio } \\
\text { D. Estándar } \\
\text { C. de Variac. } \\
\mathrm{N}\end{array}$ & $\begin{array}{c}692.7 \\
80.41 \\
11.6^{\circ} \% \\
15 \\
\end{array}$ & $\begin{array}{c}889.2 \\
109,85 \\
12.4^{\circ} \% \\
15\end{array}$ & $\begin{array}{c}116,0 \\
15,44 \\
13.3 \% \\
15\end{array}$ \\
\hline 15 & $\begin{array}{l}\text { Promedio } \\
\text { D. Estándar } \\
\text { C. de Variac. } \\
\text { N }\end{array}$ & $\begin{array}{c}721.4 \\
156.33 \\
21.7 \% \\
15\end{array}$ & $\begin{array}{c}991,1 \\
109.18 \\
11.0 \% \\
15\end{array}$ & $\begin{array}{c}145,2 \\
17,71 \\
12.2 \% \\
15\end{array}$ \\
\hline
\end{tabular}




\section{Cuadro $\mathrm{N}^{\circ} 5$}

RESULTADOS DEL ENSAYO DE FLEXIÓN ESTÁTICA CON CARGA APLICADA EN LA CARA. DIRECCIÓN DE LAS FIBRAS DE CARAS EXTERIORES, A LA LUZ.

\begin{tabular}{|c|c|c|c|c|}
\hline $\begin{array}{c}\text { ESPESOR } \\
\text { NOMINAL } \\
(\mathrm{mm})\end{array}$ & $(-)$ & $\begin{array}{l}\text { TENSION EN EL } \\
\text { LIMITE DE } \\
\text { PROPORCIONAL } \\
\left(\mathrm{kg} / \mathrm{cm}^{2}\right)\end{array}$ & $\begin{array}{l}\text { MÓDULO DE } \\
\text { RUPTURA } \\
\left(\mathrm{kg} / \mathrm{cm}^{2}\right)\end{array}$ & $\begin{array}{c}\text { MÓDULO DE } \\
\text { ELASTICIDAD } \\
\left(U \mathrm{~cm}^{2}\right)\end{array}$ \\
\hline 9 & $\begin{array}{l}\text { Promedio } \\
\text { D. Estándar } \\
\text { C. de Variac. } \\
\mathrm{N}\end{array}$ & $\begin{array}{c}721,4 \\
156,3 \\
21.7 \% \\
15 \\
\end{array}$ & $\begin{array}{c}1361,9 \\
310,4 \\
22.8 \\
15 \\
\end{array}$ & $\begin{array}{c}143,0 \\
31,54 \\
22,1 \% \\
15\end{array}$ \\
\hline 12 & $\begin{array}{l}\text { Promedio } \\
\text { D. Estándar } \\
\text { C. de Variac. } \\
\text { N }\end{array}$ & $\begin{array}{c}835,7 \\
99,11 \\
11,9 \% \\
15 \\
\end{array}$ & $\begin{array}{c}1545,0 \\
256,0 \\
16.6 \% 15\end{array}$ & $\begin{array}{c}157,3 \\
15,0 \\
9,6 \% \\
15\end{array}$ \\
\hline 15 & $\begin{array}{l}\text { Promedio } \\
\text { D. Estándar } \\
\text { C. de Variac. } \\
\text { N }\end{array}$ & $\begin{array}{c}766,0 \\
120.8 \\
15.8 \% \\
15\end{array}$ & $\begin{array}{c}1346,4 \\
164,7 \\
12,2 \% \\
15\end{array}$ & $\begin{array}{c}164,4 \\
29,1 \\
17,7 \% \\
15\end{array}$ \\
\hline
\end{tabular}

\section{Flexión Con Carga Aplicada En El Canto}

Para el desarrollo de estos ensayos se utilizaron las recomendaciones de la norma ASTM 3043-90. Además, basados en trabajos anteriores (De Urruticoechea. 1984).(Alegria y González, 1990), se agregó otra variable al estudio, ensayando probetas con distintas relaciones luz/altura. Esto último hizo aumentar el número de probetas a 162 .

A la variable $\mathbf{k}=$ luz/altura se le dieron los valores 10,14 y 18 . En este caso la luz de ensayo varia. manteniéndose constante las otras dimensiones. Al igual que el ensayo de flexión en la cara, el estudio directo de las curvas carga - deformación permitió obtener el módulo elástico, el módulo de ruptura y la tensión en el límite de proporcionalidad.

Los resultados de este ensayo se incluyen en los Cuadros $N^{\circ} 6$ y $N^{\circ} 7$

\section{Compresión}

Los ensayos se realizaron siguiendo las recomendaciones de la norma ASTM D 3501-1987 y la finalidad fue determinar la Tensión Máxima a la compresión del material en estudio. Se ensayaron un total de 90 probetas. Los resultados se incluyen en los Cuadros $\mathrm{N}^{\mathrm{D}} 8$ y N 9. 


\section{Tracción}

El objetivo de este ensayo fue determinar la Tensión Máxima. Las pruebas se realizaron siguiendo las especificaciones de la norma ASTM D 3500-90. Se ensayaron un total de 90 probetas divididas según espesor, tablero y dirección de la fibra de la cara exterior.

Las dimensiones de las probetas debieron modificarse para ajustarlas a la máquina de ensayo disponible en el laboratorio.

Cuadro $N^{\circ} 6$

RESULTADOS DEL ENSAYO DE FLEXIÓN ESTÁTICA CON CARGA APLICADA EN EL CANTO. DIRECCIÓN DE LAS FIBRAS DE CARAS EXTERIORES PARALELA A LA LUZ.

\begin{tabular}{|c|c|c|c|c|c|}
\hline $\begin{array}{c}\text { ESPESOR } \\
\text { NOMINAL } \\
(\mathrm{mm}) \\
\end{array}$ & $\begin{array}{c}\text { RAZON } \\
k=l u z / a l t . \\
(-)\end{array}$ & $\begin{array}{c}\text { ESTADIGRAFOS } \\
(-)\end{array}$ & $\begin{array}{l}\text { TENSION EN } \\
\text { EL LIMITE } \\
\left(\mathrm{kg} / \mathrm{cm}^{2}\right) \\
\end{array}$ & $\begin{array}{l}\text { MÓDULO DE } \\
\text { RUPTURA } \\
\left(\mathrm{kg} / \mathrm{cm}^{2}\right) \\
\end{array}$ & $\begin{array}{l}\text { MODULO DE } \\
\text { ELASTICIDAD } \\
\left(U \mathrm{~cm}^{2}\right)\end{array}$ \\
\hline \multirow{3}{*}{9} & 10 & $\begin{array}{l}\text { Promedio } \\
\text { D. Estándar } \\
\text { C.de Variac. }\end{array}$ & $\begin{array}{c}558.7 \\
147.8 \\
26,4 \%\end{array}$ & $\begin{array}{c}882,7 \\
223,9 \\
25,4 \%\end{array}$ & $\begin{array}{c}85,80 \\
36,72 \\
42,8 \%\end{array}$ \\
\hline & 14 & $\begin{array}{l}\text { Promedio } \\
\text { D. Estándar } \\
\text { C.de Variac. }\end{array}$ & $\begin{array}{l}604.8 \\
148.6 \\
24,6 \%\end{array}$ & $\begin{array}{c}791.1 \\
100.3 \\
12,7 \%\end{array}$ & $\begin{array}{c}80,49 \\
6,35 \\
7,9 \%\end{array}$ \\
\hline & 18 & $\begin{array}{l}\text { Promedio } \\
\text { D. Estándar } \\
\text { C.de Variac. }\end{array}$ & $\begin{array}{c}755.5 \\
95,8 \\
12,7 \%\end{array}$ & $\begin{array}{c}921.3 \\
73,5 \\
8,0 \%\end{array}$ & $\begin{array}{l}121,89 \\
51,14 \\
42,0 \%\end{array}$ \\
\hline \multirow{3}{*}{12} & 10 & $\begin{array}{l}\text { Promedio } \\
\text { D. Estándar } \\
\text { C.de Variac. }\end{array}$ & $\begin{array}{c}717,3 \\
195.9 \\
27.3 \% \\
\end{array}$ & $\begin{array}{c}844.0 \\
90.0 \\
10.6 \% \\
\end{array}$ & $\begin{array}{c}105,92 \\
25,23 \\
23.8 \% \\
\end{array}$ \\
\hline & 14 & $\begin{array}{l}\text { Promedio } \\
\text { D. Estándar } \\
\text { C.de Variac. }\end{array}$ & $\begin{array}{r}626.1 \\
131,8 \\
21,1 \%\end{array}$ & $\begin{array}{c}929,4 \\
55,2 \\
5,9 \%\end{array}$ & $\begin{array}{c}222,47 \\
37,77 \\
17,0 \%\end{array}$ \\
\hline & 18 & $\begin{array}{l}\text { Promedio } \\
\text { D. Estándar } \\
\text { C.de Variac. }\end{array}$ & $\begin{array}{c}588.1 \\
143.0 \\
24.3 \%\end{array}$ & $\begin{array}{l}909.6 \\
57.7 \\
6.3 \%\end{array}$ & \begin{tabular}{|l}
218,5 \\
64,26 \\
$29,4 \%$
\end{tabular} \\
\hline \multirow{3}{*}{15} & 10 & $\begin{array}{l}\text { Promedio } \\
\text { D. Estándar } \\
\text { C.de Variac. }\end{array}$ & $\begin{array}{c}702.9 \\
123,5 \\
17.6 \% \\
\end{array}$ & $\begin{array}{c}861,1 \\
117,6 \\
13,7 \%\end{array}$ & $\begin{array}{c}134,13 \\
63,17 \\
47,1 \%\end{array}$ \\
\hline & 14 & $\begin{array}{l}\text { Promedio } \\
\text { D. Estándar } \\
\text { C.de Variac. }\end{array}$ & $\begin{array}{c}743,1 \\
131,6 \\
17,7 \% \\
\end{array}$ & $\begin{array}{c}953,2 \\
187,4 \\
19,7 \% \\
\end{array}$ & $\begin{array}{c}209,07 \\
83,58 \\
40,0 \% \\
\end{array}$ \\
\hline & 18 & $\begin{array}{l}\text { Promedio } \\
\text { D. Estándar } \\
\text { C.de Variac. }\end{array}$ & $\begin{array}{l}531,4 \\
141.9 \\
26,7 \%\end{array}$ & $\begin{array}{c}837.7 \\
69.9 \\
8.3 \%\end{array}$ & $\begin{array}{c}161,55 \\
74,06 \\
45,8 \%\end{array}$ \\
\hline
\end{tabular}




\section{Cuadro $\mathrm{N}^{\circ} 7$}

RESULTADOS DEL ENSAYO DE FLEXIÓN ESTÁTICA CON CARGA APLICADA EN EL CANTO. DIRECCIÓN DE LAS FIBRAS DE CARAS EXTERIORES NORMAL A LA LUZ

\begin{tabular}{|c|c|c|c|c|c|}
\hline $\begin{array}{c}\text { ESPESOR } \\
\text { NOMINAL } \\
(\mathrm{mm}) \\
\end{array}$ & $\begin{array}{c}\text { RAZON } \\
k=l u z / a h t . \\
(-)\end{array}$ & $\begin{array}{c}\text { ESTADIGRAFOS } \\
(-)\end{array}$ & $\begin{array}{c}\text { TENSIÓN EN } \\
\text { EL LIMITE } \\
\left(\mathrm{kg} / \mathrm{cm}^{2}\right) \\
\end{array}$ & $\begin{array}{l}\text { MÓDULO DE } \\
\text { RUPTURA } \\
\left(\mathrm{kg} / \mathrm{cm}^{2}\right) \\
\end{array}$ & $\begin{array}{c}\text { MÓDULO DE } \\
\text { ELASTICIDAD } \\
\left(t / \mathrm{cm}^{2}\right)\end{array}$ \\
\hline \multirow{3}{*}{9} & 10 & $\begin{array}{l}\text { Promedio } \\
\text { D. Estaindar } \\
\text { C.de Variac. }\end{array}$ & $\begin{array}{c}606.3 \\
231,3 \\
38.2 \%\end{array}$ & $\begin{array}{c}807.9 \\
227.4 \\
28,1 \%\end{array}$ & $\begin{array}{c}92.68 \\
24.12 \\
26,0 \%\end{array}$ \\
\hline & 14 & $\begin{array}{l}\text { Promedio } \\
\text { D. Estaindar } \\
\text { C.de Variac. }\end{array}$ & $\begin{array}{c}664,5 \\
190,4 \\
28,6 \%\end{array}$ & $\begin{array}{c}996.9 \\
204.6 \\
20,5 \%\end{array}$ & $\begin{array}{l}229.84 \\
94.59 \\
41,2 \%\end{array}$ \\
\hline & 18 & $\begin{array}{l}\text { Promedio } \\
\text { D. Estandar } \\
\text { C.de Variac. } \\
\end{array}$ & \begin{tabular}{r|}
985.4 \\
136,4 \\
$13,8 \%$ \\
\end{tabular} & $\begin{array}{c}1113,6 \\
146,0 \\
13,1 \% \\
\end{array}$ & $\begin{array}{c}114.06 \\
15.14 \\
13,3 \% \\
\end{array}$ \\
\hline \multirow{3}{*}{12} & 10 & $\begin{array}{l}\text { Promedio } \\
\text { D. Estándar } \\
\text { C.de Variac. }\end{array}$ & $\begin{array}{c}628.9 \\
239.3 \\
38,0 \%\end{array}$ & $\begin{array}{c}1004.4 \\
99.2 \\
9.9 \%\end{array}$ & $\begin{array}{l}131,46 \\
45,433 \\
34,6 \%\end{array}$ \\
\hline & 14 & $\begin{array}{l}\text { Promedio } \\
\text { D. Estándar } \\
\text { C.de Variac. }\end{array}$ & $\begin{array}{r}604.6 \\
175,6 \\
29.0 \% \\
\end{array}$ & $\begin{array}{c}995.6 \\
100.7 \\
10,1 \% \\
\end{array}$ & $\begin{array}{c}213.54 \\
24.53 \\
11,5 \% \\
\end{array}$ \\
\hline & 18 & $\begin{array}{l}\text { Promedio } \\
\text { D. Estándar } \\
\text { C.de Variac. }\end{array}$ & $\begin{array}{l}628.7 \\
157,9 \\
25,1 \%\end{array}$ & $\begin{array}{c}1063,2 \\
169,1 \\
15,9 \%\end{array}$ & $\begin{array}{c}272.52 \\
50,37 \\
18,5 \%\end{array}$ \\
\hline \multirow{3}{*}{15} & 10 & $\begin{array}{l}\text { Promedio } \\
\text { D. Estandar } \\
\text { C.de Variac. }\end{array}$ & $\begin{array}{c}655,1 \\
132,4 \\
20,2 \% \\
\end{array}$ & $\begin{array}{c}1003.6 \\
59.1 \\
5.9 \%\end{array}$ & $\begin{array}{c}162.17 \\
65.65 \\
40.5 \% \\
\end{array}$ \\
\hline & 14 & $\begin{array}{l}\text { Promedio } \\
\text { D. Estandar } \\
\text { C.de Variac. } \\
\end{array}$ & $\begin{array}{c}746.4 \\
199.0 \\
26,7 \% \\
\end{array}$ & $\begin{array}{c}1010.2 \\
77.8 \\
7.7 \% \\
\end{array}$ & $\begin{array}{c}152.35 \\
72.92 \\
47.9 \%\end{array}$ \\
\hline & 18 & $\begin{array}{l}\text { Promedio } \\
\text { D. Estándar } \\
\text { C.de Variac. }\end{array}$ & $\begin{array}{l}675.7 \\
66.9 \\
9.9 \%\end{array}$ & $\begin{array}{l}901,1 \\
105,4 \\
11,7 \%\end{array}$ & $\begin{array}{c}149.24 \\
70.14 \\
47.0 \%\end{array}$ \\
\hline
\end{tabular}




\section{Cuadro $N^{\circ} 8$}

RESULTADOS DE LOS ENSAYOS DE COMPRESIÓN CON CARGA PARALELA A LAS FIBRAS DE LAS CARAS EXTERIORES.

\begin{tabular}{|c|l|c|}
\hline $\begin{array}{c}\text { ESPESOR NOMINAL } \\
(\mathrm{mm})\end{array}$ & \multicolumn{1}{|c|}{$\begin{array}{c}\text { ESTADiGRAFOS } \\
(-)\end{array}$} & $\begin{array}{c}\text { TENSIONES MAXIMAS } \\
\left(\mathbf{k g} / \mathrm{cm}^{2}\right)\end{array}$ \\
\hline \multirow{2}{*}{9} & Promedio & 563.2 \\
& D. Estándar & 58.5 \\
& C. de Variación & $10.4 \%$ \\
& N & 15 \\
\hline \multirow{2}{*}{12} & Promedio & 512.7 \\
& D. Estándar & 62,7 \\
& C. de Variación & $12.2 \%$ \\
& N & 15 \\
\hline \multirow{3}{*}{15} & Promedio & 571,1 \\
& D. Estandar & 59,7 \\
& C. de Variación & $10,5 \%$ \\
& N & 15 \\
\hline
\end{tabular}

Cuadro $\mathrm{N}^{\circ} 9$

RESULT ADOS DE LOS ENSAYOS DE COMPRESIÓN CON CARGA NORMAL LAS FIBRAS DE LAS CARAS FXTERIORES.

\begin{tabular}{|c|l|c|}
\hline $\begin{array}{c}\text { ESPESOR NOMINAL } \\
(\mathrm{mm})\end{array}$ & \multicolumn{1}{|c|}{$\begin{array}{c}\text { ESTADIGRAFOS } \\
(-)\end{array}$} & $\begin{array}{c}\text { TENSIONES MAXIMAS } \\
\left(\mathbf{k g} / \mathrm{cm}^{2}\right)\end{array}$ \\
\hline \multirow{3}{*}{9} & Promedio & 554.1 \\
& D. Estandar & 100.0 \\
& C. de Variación & $18.1 \%$ \\
& N & 15 \\
\hline \multirow{2}{*}{12} & Promedio & 692.0 \\
& D. Estandar & 86.7 \\
& C. de Variación & $12.5 \%$ \\
& N. & 15 \\
\hline \multirow{2}{*}{15} & Promedio & 591.9 \\
& D. Estandar & 47.5 \\
& C. de Variación & $8.0 \%$ \\
& N & 15 \\
\hline
\end{tabular}


Cuadro $\mathrm{N}^{\circ} 10$

\section{RESULTADOS DE LOS ENSAYOS DE TRACCIÓN CON CARGA PARALELA A LAS FIBRAS DE LAS CARAS EXTERIORES.}

\begin{tabular}{|c|l|c|}
\hline $\begin{array}{c}\text { ESPESOR NOMINAL } \\
(\mathrm{mm})\end{array}$ & \multicolumn{1}{|c|}{$\begin{array}{c}\text { ESTADIGRAFOS } \\
(-)\end{array}$} & $\begin{array}{c}\text { TENSIONES MAXIMAS } \\
\left(\mathrm{kg} / \mathrm{cm}^{2}\right)\end{array}$ \\
\hline \multirow{2}{*}{9} & Promedio & 565,9 \\
& D. Estándar & 68,6 \\
& C. de Variación & $12,1 \%$ \\
& N & 15 \\
\hline \multirow{2}{*}{12} & Promedio & 538,2 \\
& D. Estándar & 56.4 \\
& C. de Variación & $10,5 \%$ \\
& N & 15 \\
\hline \multirow{2}{*}{15} & Promedio & 54071,1 \\
& D. Estándar & 49,1 \\
& C. de Variación & $9,1 \%$ \\
& N & 15 \\
\hline
\end{tabular}

\section{Cuadro $\mathrm{N}^{\circ} 11$}

RESULTADOS DE LOS ENSAYOS DE TRACCIÓN CON CARGA NORMAL A LAS FIBRAS DE LAS CARAS EXTERIORES.

\begin{tabular}{|c|l|c|}
\hline $\begin{array}{c}\text { ESPESOR NOMINAL } \\
(\mathrm{mm})\end{array}$ & \multicolumn{1}{|c|}{$\begin{array}{c}\text { ESTADIGRAFOS } \\
(\mathrm{m})\end{array}$} & $\begin{array}{c}\text { TENSIONES MAXIMAS } \\
\left(\mathrm{kg} / \mathrm{cm}^{2}\right)\end{array}$ \\
\hline \multirow{2}{*}{9} & Promedio & 601.3 \\
& D. Estándar & 70.2 \\
& C. de Variación & $11.7 \%$ \\
& N & 15 \\
\hline \multirow{2}{*}{12} & Promedio & 713.9 \\
& D. Estandar & 79.2 \\
& C. de Variación & $11.1 \%$ \\
& N & 15 \\
\hline \multirow{2}{*}{15} & Promedio & 627.7 \\
& D. Estandar & 59.7 \\
& C. de Variación & $9.5 \%$ \\
& N & 15 \\
\hline
\end{tabular}

\section{Cizalle a Través del Espesor}

El objetivo de este ensayo fue determinar la Tensión Máxima de cizalle a través del espesor. El método y las dimensiones de las probetas corresponden a recomendaciones de los investigadores alemanes Mōhler y Ehlbeck (1974). Durante el ensayo. la probeta es cargada en el canto superior, distribuyendo la carga en el tercio central, mientras descansa sobre dos placas ubicadas en los tercios extremos. Los resultados obtenidos en este ensayo se muestran en los Cuadros $\mathrm{N}^{\circ} 12$ y $\mathrm{N}^{\circ} 13$ 


\section{Cuadro $N^{\circ} 12$}

RESULTADOS DE LOS ENSAYOS DE CIZALLE A TRAVES DEL ESPESOR, CON CARGA PARALELA A LAS FIBRAS DE LAS CARAS EXTERIORES, USANDO LA SECCIÓN TRANSVERSAL BRUTA

\begin{tabular}{|c|c|c|}
\hline $\begin{array}{l}\text { ESPESOR NOMINAL } \\
(\mathbf{m m})\end{array}$ & $\begin{array}{c}\text { ESTADIGRAFOS } \\
(-)\end{array}$ & $\begin{array}{l}\text { TENSIONES MAXXIMAS } \\
\left(\mathrm{kg} / \mathrm{cm}^{2}\right)\end{array}$ \\
\hline 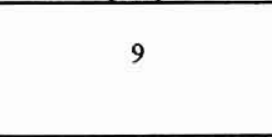 & $\begin{array}{l}\text { Promedio } \\
\text { D. Estándar } \\
\text { C. de Variación } \\
\mathrm{N}\end{array}$ & $\begin{array}{c}86,3 \\
9,4 \\
10,9 \% \\
15 \\
\end{array}$ \\
\hline 12 & $\begin{array}{l}\text { Promedio } \\
\text { D. Estándar } \\
\text { C. de Variación } \\
\mathrm{N}\end{array}$ & $\begin{array}{c}96.0 \\
16,2 \\
16,8 \% \\
15 \\
\end{array}$ \\
\hline 15 & $\begin{array}{l}\text { Promedio } \\
\text { D. Estándar } \\
\text { C. de Variación } \\
\text { N }\end{array}$ & $\begin{array}{c}98.6 \\
11,1 \\
11,3 \% \\
15\end{array}$ \\
\hline
\end{tabular}

\section{Cuadro $\mathrm{N}^{\circ} 13$}

RESULTADOS DE LOS ENSAYOS DE CIZALLE A TRAVÉS DEL ESPESOR, CON CARGA NORMAL A LAS FIBRAS DE LAS CARAS EXTERIORES, USANDO LA SECCIÓN TRANSVERSAL BRUTA.

\begin{tabular}{|c|l|c|}
\hline $\begin{array}{c}\text { ESPESOR NOMINAL } \\
(\mathrm{mm})\end{array}$ & \multicolumn{1}{|c|}{$\begin{array}{c}\text { ESTADIGRAFOS } \\
(-)\end{array}$} & $\begin{array}{c}\text { TENSIONES MAXIMAS } \\
\left(\mathrm{kg} / \mathrm{cm}^{2}\right)\end{array}$ \\
\hline \multirow{2}{*}{9} & Promedio & 106,1 \\
& D. Estándar & 9.4 \\
& C. de Variación & $8.8 \%$ \\
& N & 15 \\
\hline \multirow{2}{*}{12} & Promedio & 112,8 \\
& D. Estandar & 20.4 \\
& C. de Variación & $18,1 \%$ \\
& N. & 13 \\
\hline \multirow{2}{*}{15} & Promedio & 119.8 \\
& D. Estándar & 15,0 \\
& C. de Variación & $12,5 \%$ \\
& N & 15 \\
\hline
\end{tabular}

Cizalle Interlaminar o a través del Espesor, en un Plano Paralelo a las Caras Exteriores

El propósito del ensayo fue determinar la Tensión Máxima de cizalle interlaminar. Los ensayos se ciñeron a las indicaciones de la norma ASTM D 2718-90. 
Para realizar las pruebas, una probeta de forma rectangular es adherida por sus caras con un adhesivo epóxico entre dos placas de acero, las que tienen uno de sus bordes afilados. Estos se proyectan más allá del extremo de la probeta. El ensayo se realiza cargando los bordes afilados con compresión, aumentando la carga en forma gradual. Es importante destacar que las probetas sólo se ensayan con la fibra de la cara exterior perpendicular a la dirección de la carga. Los resultados de este ensayo se presentan en el Cuadro $\mathrm{N}^{\circ} 14$.

\section{Cuadro $\mathrm{N}^{\circ} 14$}

RESULTADOS DE LOS ENSAYOS DE CIZALLE INTERLAMINAR CON CARGA NORMAL A LAS FIBRAS DE LAS CARAS EXTERIORES, USANDO LA SECCIÓN TRANSVERSAL BRUTA.

\begin{tabular}{|c|c|c|}
\hline $\begin{array}{l}\text { ESPESOR NOMINAL } \\
(\mathrm{mm})\end{array}$ & $\begin{array}{c}\text { ESTADÍGRAFOS } \\
(-)\end{array}$ & $\begin{array}{l}\text { TENSIONES MÁXIMAS } \\
\left(\mathrm{kg} / \mathrm{cm}^{2}\right)\end{array}$ \\
\hline 9 & $\begin{array}{l}\text { Promedio } \\
\text { D. Estándar } \\
\text { C. de Variación } \\
\mathrm{N}\end{array}$ & $\begin{array}{c}33.0 \\
4.7 \\
14.2 \% \\
15 \\
\end{array}$ \\
\hline 12 & $\begin{array}{l}\text { Promedio } \\
\text { D.Estándar } \\
\text { C. de Variación } \\
\mathrm{N}\end{array}$ & $\begin{array}{c}24.2 \\
6.7 \\
27.7 \% \\
15 \\
\end{array}$ \\
\hline 15 & $\begin{array}{l}\text { Promedio } \\
\text { D. Estándar } \\
\text { C. de Variación } \\
\mathrm{N}\end{array}$ & $\begin{array}{c}27,1 \\
2,1 \\
7.9 \% \\
15 \\
\end{array}$ \\
\hline
\end{tabular}

\section{Módulo de Cizalle en el Plano}

Para determinar el Módulo de Corte de materiales ortotrópicos. como el contrachapado, existe un método de ensayo en la norma ASTM 3044-86. La ventaja de este procedimiento es que permite conocer el Módulo de Corte en el plano en forma directa, sin necesidad de conocer el Módulo Elástico.

El procedimiento consiste en torcer una placa cuadrada del material, aplicando la carga en dos vértices opuestos y sujetándola en los otros dos. Es necesario registrar en cada instante la carga aplicada y la deformación relativa que sufren las dos diagonales de la placa.

Los resultados obtenidos en dicho ensayo se incluyen en el Cuadro $N^{\circ} 15$. 
Cuadro $N^{\circ} 15$

RESULTADOS DE LOS ENSAYOS DESTINADOS A DETERMINAR EL MÓDULO DE CORTE (G).

\begin{tabular}{|c|c|c|}
\hline $\begin{array}{c}\text { ESPESOR } \\
\text { NOMINAL } \\
(\mathrm{mm}) \\
\end{array}$ & $\begin{array}{c}\text { ESTADIGGAFOS } \\
(\cdot) \\
\end{array}$ & $\begin{array}{c}\text { VALOR } \\
\text { MAXIMO } \\
\left(\mathrm{kg}^{\prime} \mathrm{cm}^{2}\right) \\
\end{array}$ \\
\hline 9 & $\begin{array}{l}\text { Promedio } \\
\text { D. Estándar } \\
\text { Coef. de Variación }\end{array}$ & $\begin{array}{c}12119.4 \\
1066,5 \\
8,8 \%\end{array}$ \\
\hline 12 & $\begin{array}{l}\text { Promedio } \\
\text { D. Estándar } \\
\text { Coef. de Variación }\end{array}$ & $\begin{array}{l}6217,2 \\
300,8 \\
4,8 \% \\
\end{array}$ \\
\hline 15 & $\begin{array}{l}\text { Promedio } \\
\text { D. Estándar } \\
\text { Coef. de Variación }\end{array}$ & $\begin{array}{c}5402,1 \\
305,6 \\
5,7 \%\end{array}$ \\
\hline
\end{tabular}

\section{Tensiones Básicas del Contrachapado de Coigüe}

Para Secciones Transversales Efectivas y Transformadas.

Se elegirá el método adoptado por la American Plywood Association (APA). Ella deduce las Tensiones Básicas del contrachapado considerando el valor mínimo. calculado con una confiabilidad del $95 \%$ ( $\mathbf{R}_{\text {min.5.5\% }}$ ), para las propiedades resistentes. $\mathbf{y}$ el valor promedio para las caracteristicas elásticas determinadas en el laboratorio.

Los factores de ajuste (n), que refieren las propiedades establecidas de los ensayos de corta duración a una duración acumulada total de la carga de 10 años, (duración "normal"), resultan los mismos que los usados para la madera aserrada (NCh 1198). Sin embargo. los factores de seguridad inherentes a efectos de fabricación y utilización no pueden ser los mismos. especialmente para aquellas características del contrachapado que dependen en gran medida del proceso de elaboración, como son las propiedades de resistencia al cizalle.

Las Tensiones Básicas $\left(\mathrm{F}_{\mathrm{b}}\right)$ quedan definidas. entonces, por la relación :

$$
F_{b}=\frac{R_{\min } 5 \%}{n}\left(\mathrm{~kg} / \mathrm{cm}^{2}\right)
$$

En el Cuadro $N^{\circ} 16$ se indican los Factores de Ajuste considerados por la APA en el proceso de determinación de las Tensiones Básicas de la madera natural, aplicables al contrachapado. 


\section{Cuadro $\mathrm{N}^{\circ} 16$}

FACTORES DE AJUSTE TOTAL.

\begin{tabular}{|l|c|c|c|}
\hline $\begin{array}{c}\text { PROPIEDAD DE LA } \\
\text { MADERA ASERRADA }\end{array}$ & $\begin{array}{c}\text { FACTOR DE MODIFICACIÓN } \\
\text { POR DURACIÓN DE CARGA }\end{array}$ & $\begin{array}{c}\text { COEFICIENTE DE } \\
\text { SEGURIDAD TOTAL }\end{array}$ & $\begin{array}{c}\text { FACTOR DE } \\
\text { AJUTE TOTAL }\end{array}$ \\
\hline Flexión yTracción & 1,6 & 1,4 & 2,24 \\
\hline Compresión en elcanto & 1,5 & 1,7 & 2,55 \\
\hline Cizalle & 1,6 & 2,8 & 4,48 \\
\hline Compresión normal & - & $\cdot$ & $\cdot$ \\
\hline Módulo de Corte & - & - & E/20 \\
\hline
\end{tabular}

Cabe hacer notar que el módulo de elasticidad aparente en flexión estática $\left(E_{f}\right)$, se deduce de ensayos realizados en probetas estándar ASTM de madera aserrada, para la relación:

$$
\begin{gathered}
\text { luz/altura }=1 / \mathrm{h}=14 \\
\text { y su valor es } 105500\left(\mathrm{~kg} / \mathrm{cm}^{2}\right)
\end{gathered}
$$

El módulo elástico asi determinado incorpora implícitamente las deformaciones por corte en la expresión que entrega la deformación por flexión, cuya magnitud, para relaciones $E / G=20$, ascienden aproximadamente al $10 \%$ de las deformaciones originadas por la flexión. En consecuencia. el $E_{f}$ tenderá a subestimar las deformaciones de elementos con relaciones $1 / \mathrm{h}<14$ y a sobre estimarlas, en el caso contrario. En las aplicaciones habituales resulta $1 / \mathrm{h}>14$, por lo que resulta práctico y conservador usar el módulo elástico aparente, deducido de los ensayos de flexión en madera aserrada. Cuando $1 / \mathrm{h}<14$, convendrá usar el $\mathrm{E}_{\mathrm{f}}$ aparente incrementado en un $10 \%$ y evaluar las deformaciones por flexión y corte en forma separada, mediante las relaciones habituales de la Resistencia de Materiales.

Para los usos normales del contrachapado, como cubierta de piso o techos. donde el material queda sometido a flexión en su plano, se puede estimar la Tensión Básica a cizalle interlaminar como:

$$
F_{b, c z, i}=\frac{F_{b, c z}}{2,5} \quad\left(\mathrm{~kg} / \mathrm{cm}^{2}\right)
$$

Cuando se prevean concentraciones de tensiones, como por ejemplo en la superficie de contacto entre alma y alas de una viga compuesta con alma de contrachapado, o bien en los bordes exteriores de paneles nervados, la APA recomienda reducir dicha tensión en un $50 \%$. 
De esta forma resulta que las Tensiones Básicas del coigüe son las incluídas en el Cuadro N¹7.

\section{Cuadro No 17}

TENSIONES BASICAS DEL CONTRACHAPADO DE COIGÖE, DETERMINADAS CON LA SECCIÓN TRANSVERSAL EFECTIVA.

\begin{tabular}{|c|c|c|c|c|c|c|}
\hline \multicolumn{2}{|c|}{$\begin{array}{c}\text { ENSAYO O } \\
\text { SOLICITACIÓN* } \\
(-) \\
\end{array}$} & $\begin{array}{c}\text { ESPESOR } \\
\text { NOMINAL } \\
(\mathrm{mm})\end{array}$ & $\begin{array}{l}R_{\operatorname{man}} \\
\left(\mathrm{kg} / \mathrm{cm}^{2}\right)\end{array}$ & $\begin{array}{l}\text { FACTOR } \\
\text { DE } \\
\text { AUUSTE } \\
(\cdot)\end{array}$ & $\begin{array}{l}\text { TENSIÓN } \\
\text { BÁSICA } \\
\left(\mathrm{kg} / \mathrm{cm}^{2}\right)\end{array}$ & OBSERVACIONES \\
\hline \multirow{9}{*}{$\begin{array}{l}\text { FLEXION } \\
\text { CON } \\
\text { CARGA } \\
\text { APLICADA } \\
\text { EN LA } \\
\text { CARA }\end{array}$} & Paralel & \multirow[t]{3}{*}{ 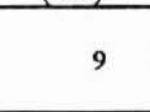 } & 661,95 & \multirow{3}{*}{2,24} & 295,5 & Valor minimo \\
\hline & & & & & & \\
\hline & Normal & & 851,29 & & 380,0 & \\
\hline & Paralel & \multirow{3}{*}{12} & 708,40 & \multirow{3}{*}{2,24} & 316,3 & \\
\hline & & & & & & \\
\hline & Normal & & 903,57 & & 403,4 & \\
\hline & Paralel & \multirow{3}{*}{15} & 811,50 & \multirow{3}{*}{2,24} & 362,3 & \\
\hline & & & & & & \\
\hline & Normal & & 1075,47 & & 480,1 & Valor máximo \\
\hline \multirow{9}{*}{$\begin{array}{l}\text { FLEXION } \\
\text { CON } \\
\text { CARGA } \\
\text { APLICADA } \\
\text { EN EL } \\
\text { CANTO } \\
\text { ** }\end{array}$} & Paralel & \multirow{3}{*}{9} & 626,11 & \multirow{3}{*}{2.24} & 279,5 & Valor minimo \\
\hline & & & & & & \\
\hline & Normal & & 660,33 & & 294,8 & \\
\hline & Paralel & \multirow{3}{*}{12} & 838,60 & \multirow{3}{*}{2,24} & 374,4 & \\
\hline & & & & & & \\
\hline & Normal & & 829,95 & & 370,5 & \\
\hline & Paralel & \multirow{3}{*}{15} & 644,93 & \multirow{3}{*}{2.24} & 287,9 & \\
\hline & & & & & & \\
\hline & Normal & & 882,22 & & 393,8 & Valor máximo \\
\hline \multirow{9}{*}{$\begin{array}{l}\text { COMPRE- } \\
\text { SION } \\
\text { CON } \\
\text { CARGA } \\
\text { APLICADA } \\
\text { EN EL } \\
\text { CANTO }\end{array}$} & Paralel & \multirow{3}{*}{9} & 466,97 & \multirow{3}{*}{2,55} & 183,1 & \\
\hline & & & & & & \\
\hline & Normal & & 389,69 & & 152,8 & Valor mínimo \\
\hline & Paralel & \multirow{3}{*}{12} & 409,56 & \multirow{3}{*}{2.55} & 160,6 & \\
\hline & & & & & & \\
\hline & Normal & & 549,38 & & 215,4 & Valor máximo \\
\hline & Paralel & \multirow{3}{*}{15} & 472.89 & \multirow{3}{*}{2.55} & 185,4 & \\
\hline & & & & & & \\
\hline & Normal & & 513,76 & & 201.5 & \\
\hline \multirow{8}{*}{$\begin{array}{l}\text { TRACCION } \\
\text { CON } \\
\text { CARGA } \\
\text { APLICADA } \\
\text { EN EL } \\
\text { CANTO }\end{array}$} & Paralel & \multirow{3}{*}{9} & 453,05 & \multirow{3}{*}{2.24} & 202,2 & \\
\hline & & & & & & \\
\hline & Normal & & 485,82 & & 216,9 & \\
\hline & Paralel & \multirow{3}{*}{12} & 445,42 & \multirow{3}{*}{2,24} & 198,8 & Valor minimo \\
\hline & & & & & & \\
\hline & Normal & & 583,62 & & 260,5 & Valor máximo \\
\hline & Paralel & \multirow[b]{2}{*}{15} & 459,33 & & 205,3 & \\
\hline & Normal & & 529.49 & 2.24 & 236.4 & \\
\hline
\end{tabular}




\section{Cuadro $N^{\circ} 17$ (Continuación)}

\begin{tabular}{|c|c|c|c|c|c|c|}
\hline \multicolumn{2}{|c|}{$\begin{array}{c}\text { ENSAYOO } \\
\text { SOLICITACIÓN* } \\
(-)\end{array}$} & $\begin{array}{c}\text { ESPESOR } \\
\text { NOMINAL } \\
(\mathrm{mm})\end{array}$ & $\begin{array}{c}R_{\operatorname{mm} s \times} \\
\left(\mathrm{kg} / \mathrm{cm}^{2}\right)\end{array}$ & $\begin{array}{l}\text { FACTOR } \\
\text { DE } \\
\text { AJUSTE } \\
(-)\end{array}$ & $\begin{array}{l}\text { TENSIÓN } \\
\text { BÁSICA } \\
\left(\mathrm{kg} / \mathrm{cm}^{2}\right) \\
\end{array}$ & OBSERVACIONES \\
\hline \multirow{7}{*}{$\begin{array}{l}\text { CIZALLE A } \\
\text { TRAVES } \\
\text { DEL } \\
\text { ESPESOR }\end{array}$} & Paralel & & 70,84 & & 15,8 & \\
\hline & & 9 & & 4,48 & & \\
\hline & Normal & & 90,64 & & 20,2 & \\
\hline & Paralel & \multirow[b]{2}{*}{12} & 69,35 & \multirow[b]{2}{*}{4,48} & 15,5 & Valor mínimo \\
\hline & Normal & & 79,24 & & 17,7 & \\
\hline & Paralel & \multirow[b]{2}{*}{15} & 80,34 & \multirow[b]{2}{*}{4,48} & 17,9 & \\
\hline & Normal & & 95,13 & & 21,2 & Valor máximo \\
\hline $\begin{array}{l}\text { CIZALLE } \\
\text { INTERLA- } \\
\text { MINAR }\end{array}$ & Normal & $\begin{array}{r}9 \\
12 \\
15 \\
\end{array}$ & $\begin{array}{l}25,27 \\
14,37 \\
23,65 \\
\end{array}$ & $\begin{array}{l}2,5 \\
2,5 \\
2,5 \\
\end{array}$ & $\begin{array}{r}10,1 \\
5,7 \\
9,5 \\
\end{array}$ & $\begin{array}{l}\text { Valor máximo } \\
\text { Valor minimo }\end{array}$ \\
\hline \multirow{6}{*}{$\begin{array}{l}\text { MODULO } \\
\text { ELASTICO } \\
\text { FLEXION } \\
\text { CON CARGA } \\
\text { APLICADA } \\
\text { EN LA } \\
\text { CARA }\end{array}$} & Paralel & \multirow[b]{2}{*}{9} & 132700 & \multirow[b]{2}{*}{1,0} & 132700 & \\
\hline & Normal & & 143000 & & 143000 & \\
\hline & Paralel & \multirow[b]{2}{*}{12} & 116000 & \multirow[b]{2}{*}{1,0} & 116000 & Valor minimo \\
\hline & Normal & & 147000 & & 147000 & \\
\hline & Paralel & \multirow[b]{2}{*}{15} & 145200 & \multirow[b]{2}{*}{1,0} & 145200 & \\
\hline & Normal & & 164200 & & 164200 & Valor máximo \\
\hline \multirow{7}{*}{$\begin{array}{l}\text { MÓDULO } \\
\text { ELÁSTICO } \\
\text { FLEXIÓN } \\
\text { CON CARGA } \\
\text { APLICADA } \\
\text { EN EL } \\
\text { CANTO }\end{array}$} & Paralel & \multirow[b]{2}{*}{9} & 80490 & \multirow[b]{2}{*}{1,0} & 80490 & Valor minimo \\
\hline & Normal & & 229840 & & 229840 & \\
\hline & Paralel & \multirow{3}{*}{12} & 222470 & \multirow{3}{*}{1.0} & 222470 & \\
\hline & & & & & & \\
\hline & Normal & & 213540 & & 213540 & \\
\hline & Paralel & \multirow[b]{2}{*}{15} & 290070 & \multirow[b]{2}{*}{1,0} & 290070 & Valor máximo \\
\hline & Normal & & 152350 & & $152350^{\circ}$ & \\
\hline \multirow{3}{*}{\multicolumn{2}{|c|}{$\begin{array}{l}\text { MÓDULO } \\
\text { DE } \\
\text { (G) }\end{array}$}} & 9 & 10365 & 1.0 & 10365 & Valor máximo \\
\hline & & 12 & 5722 & 1,0 & 5722 & \\
\hline & & 15 & 4899 & 1,0 & 4899 & Valor minimo \\
\hline
\end{tabular}

(*) Paralel : Fibra cara exterior paralela a la luz.

Normal : Fibra cara exterior perpendicular a la luz.

(*) Razón luz/altura $=1 / \mathrm{h}=14$

\section{Razones de Resistencia}

Los máximos defectos permitidos para el peor nivel de chapas que conforman cada tablero afectarán adicionalmente sus propiedades resistentes. La incorporación de este 
efecto permite evaluar las tensiones admisibles tomando como parámetro las razones de resistencia.

Los factores de reducción de la resistencia por defectos permitidos. aplicados por la APA. se incluyen en el Cuadro $\mathrm{N}^{\circ} 18$.

Cuadro $\mathrm{N}^{\circ} 18$

RAZONES DE RESISTENCIAS APLICADAS.

\begin{tabular}{|l|c|c|c|}
\hline \multirow{2}{*}{ PROPIEDAD } & \multicolumn{3}{|c|}{ NIVEL DE TENSIONES } \\
\cline { 2 - 4 } & S1 & S2 & S3 \\
\hline FLEXIÓN & 0,60 & 0,50 & 0,50 \\
\hline COMPRESIÓN & 0,80 & 0,75 & 0,75 \\
\hline TRACCIÓN & 0,60 & 0,50 & 0,50 \\
\hline CIZALLE & 1,00 & 1,00 & 0,85 \\
\hline CIZALLE INTERLAMINAR & 1,00 & 1,00 & 0,90 \\
\hline COMPRESIÓN NORMAL & 1,00 & 1.00 & 1,00 \\
\hline MÓDULOS ELÁSTICOS & 1,00 & 1,00 & 1,00 \\
\hline
\end{tabular}

El nivel S1 es aplicable a todo contrachapado compuesto por tulipas N, A ó C y el nivel S2 a las chapas B, C taponeado ó D. encoladas con adhesivo tipo exterior.

El nivel S3 es aplicable a los contrachapados de tulipas B, C taponeado y D, encoladas con adhesivo tipo interior y por lo tanto. no aptos para ambientes húmedos, ni para tableros contrachapados estructurales.

El caso que se analiza (coigüe) corresponde a un nivel de tensiones S2 pues, se consideraron chapas tipo $\mathrm{C}$ y D. encoladas con adhesivo tipo exterior.

\section{Tensiones Admisibles}

En el Cuadro $N^{\circ} 19$ se incluye el cálculo de las Tensiones Admisibles, para el Estado Seco $(\mathrm{H}<16 \%)$. es decir, cuando se espera que el contrachapado no supere en servicio, en forma continua o permanente, una humedad propia de un $16 \%$. 


\section{Cuadro $\mathrm{N}^{\circ} 19$}

\section{VALORFS DE LAS TENSIONES ADMISIBLES CALCULADAS PARA EL CONTRACHAPADO DE COIGOEE}

\begin{tabular}{|c|c|c|c|c|c|}
\hline \multirow[t]{3}{*}{ PROPIEDAD } & \multicolumn{3}{|c|}{ TENSIONES BÁSICAS } & \multirow{3}{*}{$\begin{array}{c}\text { RAZON DE } \\
\text { RESISTENCIA } \\
(-)\end{array}$} & \multirow{3}{*}{$\begin{array}{c}\text { TENSION } \\
\text { ADMISIBLE } \\
\left(\mathrm{kg} / \mathrm{cm}^{2}\right)\end{array}$} \\
\hline & CALCULAD & PROPUEST & ELEGIDA & & \\
\hline & $\left(\mathrm{kg} / \mathrm{cm}^{2}\right)$ & $\left(\mathrm{kg} / \mathrm{cm}^{2}\right)$ & $\left(\mathrm{kg} / \mathrm{cm}^{2}\right)$ & & \\
\hline FLEXIÓN & 279,5 & 280,0 & 280,0 & 0,50 & 140.0 \\
\hline COMPRESIÓN & 152,8 & 152,8 & 152,8 & 0,75 & 115,0 \\
\hline TRACCION & 198,8 & 200,0 & 200,0 & 0,50 & 100.0 \\
\hline CIZALLE & 15,5 & 15,5 & 15,5 & 1,00 & 15,5 \\
\hline $\begin{array}{l}\text { CIZALLE } \\
\text { INTERLAMIN }\end{array}$ & 5,7 & $6,2(1)$ & 6,2 & 1,00 & 6,2 \\
\hline Ef & 116000 & $\begin{array}{c}105500 \\
\text { (2) }\end{array}$ & 105500 & 1.00 & 105500 \\
\hline $\bar{G}$ & 4899 & 5275 & $\begin{array}{c}5275 \\
(3)\end{array}$ & 1,00 & 5275 \\
\hline
\end{tabular}

NOTAS: (1) Estimado como el valor básico para el cizalle a través del espesor, dividido por el factor 2,5 para usos normales como pisos, cielos ,techumbres, es decir, con solicitaciones de flexión en su plano. (Procedimiento de la APA)

(2) Estimado como el módulo de elasticidad en flexión. determinado en madera natural de coigúe. usando la probeta estándar ASTM, en estado seco (APA).

(3) Estimado como $E_{1} / 20$, segun recomendaciones de la APA

Estas Tensiones Admisibles se aplican al contrachapado de coigüe, fabricado por BOMASA, en estado seco $(\mathrm{H}<16 \%$ ), para cargas normales (duración 10 años). sin tratamiento de ignifugos.

\section{Factores de Modificación de Aplicación General}

Si las condiciones de servicio son distintas a las señaladas anteriormente, se deberá recurrir a los Factores de Modificación de Aplicación General, los que deben considerar las reales condiciones de uso y servicio del tablero.

\section{Factor de Modificación por Duración de Carga $\left(K_{\mathrm{D}}\right)$}

El valor del factor de modificación por duración de carga $\left(\mathrm{K}_{\mathrm{D}}\right)$, se obtiene de la expresión :

$$
K_{D}=\frac{1,747}{t^{0,0464}}+0,295
$$

donde: $t=$ duración de la carga, en segundos. 


\section{Factor de Modificación por Humedad $\left(\mathbf{K}_{\mathbf{t}}\right)$.}

Si el contrachapado tendrá una humedad de servicio igual o mayor que $16 \%$ ( $\mathrm{H}>$ $16 \%$ ), el factor de modificación se obtiene del Cuadro $N^{\circ} 20$.

\section{Factor de Modificación por Tratamiento Químico.}

Para los contrachapados sometidos a tratamientos de preservantes a vacío y presión, no es necesaria una modificación de las tensiones admisibles. Sin embargo, para los tratamientos con productos ignífugos, se recomienda reducir las tensiones admisibles en un $16.7 \%$ y las propiedades elásticas en un $10.0 \%$.

\section{Cuadro $\mathrm{N}^{\circ} 20$}

\section{FACTORES DE MODIFICACIÓN POR HUMEDAD PARA H $>16 \%$}

\begin{tabular}{|l|c|}
\hline PROPIEDAD & $\begin{array}{c}\text { F. DE M. POR HUMEDAD } \\
\mathrm{K}_{\mathrm{H}}\end{array}$ \\
\hline FLEXIÓN & 0,714 \\
\hline COMPRESIÓN & 0,588 \\
\hline TRACCIÓN & 0.714 \\
\hline CIZALLE & 0,813 \\
\hline CIZALLE INTERLAMINAR & 0,840 \\
\hline MÓDULO DE ELASTICIDAD & 0,833 \\
E $_{1}$ & \\
\hline MÓDULO DE CORTE & 0,800 \\
\hline
\end{tabular}

\section{CONCLUSIONES}

1.- El contenido de humedad que tenian los tableros en el momento de ser ensayados era de $8.7 \%$ para los tableros con espesor de $9 \mathrm{~mm}$, de $9.0 \%$ para el espesor de $12 \mathrm{~mm}$ y de $9.7 \%$ para el de $15 \mathrm{~mm}$.

2.- La densidad de referencia de los tableros ensayados resultó de $483 \mathrm{~kg} / \mathrm{m}^{3}$ para el espesor de $9 \mathrm{~mm}$. de $523 \mathrm{~kg} / \mathrm{m}^{3}$ para el de $12 \mathrm{~mm}$ y de $650 \mathrm{~kg} / \mathrm{m}^{3}$ para el de $15 \mathrm{~mm}$.

3.- El peso por unidad de superficie de los tableros ensayados resultó ser de $4,7 \mathrm{~kg} / \mathrm{m}^{2}$ para el tablero de $9 \mathrm{~mm}$ de espesor, de $6.6 \mathrm{~kg} / \mathrm{m}^{2}$ para el tablero de $12 \mathrm{~mm}$ de espesor y de $10.0 \mathrm{~kg} / \mathrm{m}^{2}$ para el de $15 \mathrm{~mm}$ de espesor.

4.- Las propiedades geométricas que se obtienen al considerar la Sección Transversal Efectiva del contrachapado, es decir al tomar como secciones efectivas de una sección transversal sólo a las chapas cuya fibras tienen la misma dirección de los esfuerzos principales. se incluyen en el Cuadro siguiente. 


\section{Cuadro $\mathrm{N}^{\circ} 21$}

PROPIEDADES GEOMETRICAS DEL CONTRACHAPADO DE COIGOE PARA USO ESTRUCTURAL. CONSIDERANDO EL METODO DE LA SECCION TRANSVERSAL EFECTIVA.

\begin{tabular}{|c|c|c|c|c|c|c|c|c|c|c|}
\hline \multirow{2}{*}{$\begin{array}{l}\text { Epeseor } \\
\text { nominal } \\
\text { (min) }\end{array}$} & \multirow{2}{*}{ 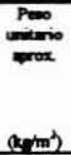 } & \multirow{2}{*}{$\begin{array}{l}\text { Equesor } \\
\text { dectivo } \\
\text { (num) }\end{array}$} & \multicolumn{4}{|c|}{ 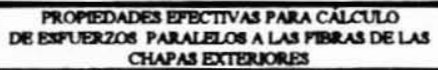 } & \multicolumn{4}{|c|}{ 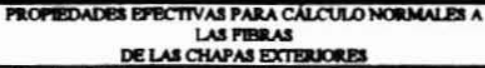 } \\
\hline & & & $\underset{\left(\mathrm{cm}^{2}\right)}{\mathrm{A}}$ & $\begin{array}{l}\text { Momasio } \\
\text { Ae harcia } \\
\text { I } \\
\text { (cin') }\end{array}$ & $\begin{array}{l}\text { Modulo } \\
\text { a vecribn } \\
\text { W } \\
\left(\mathrm{cm}^{3}\right)\end{array}$ & $\begin{array}{l}\text { Ce paried conte } \\
\text { intertaminim } \\
\text { b.vs } \\
\text { (cm) }\end{array}$ & $\underset{\left(\mathrm{cm}^{2}\right)}{\text { Ares de }}$ & $\begin{array}{c}\text { Momariode } \\
\text { Inercia } \\
1 \\
\left(\mathrm{~cm}^{2}\right)\end{array}$ & $\begin{array}{l}\text { Modulo it } \\
\text { meccion } \\
\text { w/ } \\
\text { (cm) }\end{array}$ & $\begin{array}{l}\text { Ce pend corte } \\
\text { intertemi } \\
\text { b.ys } \\
\left(\mathrm{cm}^{2}\right)\end{array}$ \\
\hline (1) & (2) & (3) & (4) & (5) & (6) & (7) & (8) & (9) & (10) & (11) \\
\hline 9 & 4,72 & 9,30 & 60,00 & 3,14 & 11,05 & 61,97 & 33,00 & 1,57 & 5,31 & 39,31 \\
\hline 12 & 6.59 & 12,50 & 71.60 & 11,18 & 18,17 & 82,02 & 51,39 & 4,33 & 9,93 & 58,13 \\
\hline 15 & 9.97 & 15,40 & 85,50 & 20,24 & 26,29 & 102.66 & 68,40 & 10,19 & $17, \infty$ & 79.88 \\
\hline
\end{tabular}

NOTA: Los valores indicados en las columnas (4) a (11) se refieren a un ancho de $100 \mathrm{~cm}$.

5.- Las Tensiones Admisibles aplicables al contrachapado estructural seco $(\mathrm{H}<16 \%)$, de coigüe, fabricado por BOMASA, se presentan en el Cuadro siguiente.

Cuadro $\mathrm{N}^{\circ} 22$

TENSIONES ADMISIBLES DEL CONTRACHAPADO ESTRUCTURAL DE COIGÓE

\begin{tabular}{|l|c|}
\hline PROPIEDAD & $\begin{array}{c}\text { TENSIÓN } \\
\text { ADMISIBLE } \\
\mathrm{kg} / \mathrm{cm}^{2}\end{array}$ \\
\hline FLEXIÓN & 140,0 \\
COMPRESIÓN & 115,0 \\
TRACCIÓN & 100,0 \\
CIZALLE A TRAVÉS DEL ESPESOR & 15,5 \\
CIZALLE INTERLAMINAR & 6,2 \\
MÓDULO DE ELASTICIDAD & 105500 \\
MÓDULO DE CORTE & 5275 \\
\hline
\end{tabular}

6.- De acuerdo a la denominación definida en la norma chilena NCh 1198 (Tablas 3a y $3 \mathrm{~b}$ ), estos valores se aproximan bastante a la Clase Estructural $\mathrm{F} \mathrm{14.} \mathrm{Al} \mathrm{comparar} \mathrm{los}$ valores normalizados con los obtenidos en este estudio, resulta :

\section{Cuadro $\mathrm{N}^{\circ} 23$}

TENSIONES ADMISIBLES PARA CONTRACHAPADO ESTRUCTURAL

\section{DE COIGOE Y NCh 1198}

\begin{tabular}{|l|c|c|}
\hline \multirow{2}{*}{ PROPIEDAD } & \multicolumn{2}{|c|}{ TENSIONES ADMISIBLES(MPa) } \\
\cline { 2 - 3 } & NCh 1198 & COIGÜE \\
\hline FLEXIÓN & 14,0 & 14,0 \\
\hline COMPRESIÓN & 10,5 & 11,5 \\
\hline TRACCIÓN & 11,0 & 10,0 \\
\hline CIZALLE A TRAVÉS DELESPESOR & - & 15,5 \\
\hline CIZALLE INTERLAMINAR & 2,1 & 6,2 \\
\hline MÓDULO DE ELASTICIDAD & 9.100 & 10.550 \\
\hline MÓDULO DE CORTE & 455 & 527,5 \\
\hline
\end{tabular}

- Valores obtenidos en el presente estudio. 
7.- El contrachapado estructural de coigie, fabricado por BOMASA, seco $(\mathrm{H}<16 \%)$, se puede asimilar a la Clase Estructural F 14, definida en la norma chilena NCh 1198.

\section{REFERENCIAS BIBLIOGRÁFICAS.}

ALEGRIA M. y GONZALEZ S.,1990. Estudio de las Propiedades Mecánicas del Contrachapado Estructural INFODEMA S.A. Memoria para optar al título de Ingeniero Civil en Obras Civiles, Facultad de Ingenieria, Universidad de Santiago de Chile, Santiago.

AMERICAN SOCIETY FOR TESTING AND MATERIALS, 1987. ASTM D-3043 Standard Test Method for Structural Panels in Flexure.

AMERICAN SOCIETY FOR TESTING AND MATERIALS, 1987. ASTM D-3501 Standard Methods of Testing Plywood in Compression.

AMERICAN SOCIETY FOR TESTING AND MATERIALS, 1990. ASTM D-3500 Standard Methods of Testing Structural Panels in Tension.

AMERICAN SOCIETY FOR TESTING AND MATERIALS, 1990. ASTM D-2718 Standard Test Method for Structural Panels in Planar Shear (Rolling Shear).

AMERICAN SOCIETY FOR TESTING AND MATERIALS, 1986. ASTM D-3044 Standard Test Method for Shear Modulus of Plywwod.

AMERICAN PLYWOOD ASSOCIATION, APA, Plywood Design Specifications y Anexos 1, 2,3 y 4 .

INSTITUTO NACIONAL DE NORMALIZACION, INN, 1990. NCh 1198 : MaderaConstrucciones en Madera-Cálculo, Santiago, Chile.

MÖHLER Y EHLBECK, 1974. Kurzzeit-U. Daverstandversuche fur ermittlung der statischen und daverstandfestigkeit von bau-furnierplatten. Berichte aus der bauforschung, heft 92 . Wilhelm Ernst \& Sohn.

PEREZ GALAZ VICENTE, 1995. El Contrachapado Estructural., Ciencia e Investigación Forestal, Vol.9, $\mathrm{N}^{\circ} .1$, (págs. 123 - 140)., Santiago, Chile.

RESIDENCIAL \& COMMERCIAL, 1982. APA Design/Construction, Guide N E.308.

STANDARDS ASSOCIATION OF AUSTRALIA, 1979. AS 2269, Structural Plywood 